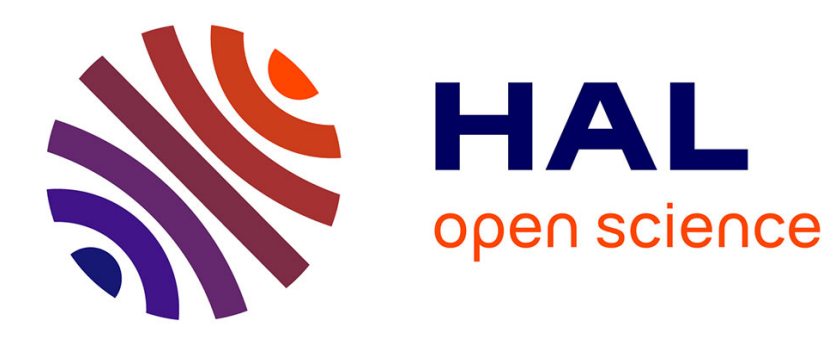

\title{
L'autochtonie dans les aires marines protégées.
}

Abdel Wedoud Ould Cheikh, Tarik Dahou

\section{To cite this version:}

Abdel Wedoud Ould Cheikh, Tarik Dahou. L'autochtonie dans les aires marines protégées.. Politique africaine, 2007, 4 (108), pp.173 à 190. hal-02268727

\section{HAL Id: hal-02268727 \\ https://hal.science/hal-02268727}

Submitted on 8 Sep 2019

HAL is a multi-disciplinary open access archive for the deposit and dissemination of scientific research documents, whether they are published or not. The documents may come from teaching and research institutions in France or abroad, or from public or private research centers.
L'archive ouverte pluridisciplinaire HAL, est destinée au dépôt et à la diffusion de documents scientifiques de niveau recherche, publiés ou non, émanant des établissements d'enseignement et de recherche français ou étrangers, des laboratoires publics ou privés. 
Tarik dahou et Abdel Wedoud Ould Cheikh

\title{
L'AUTOCHTONIE DANS LES AIRES MARINES PROTÉGÉES. Terrain de conflit en Mauritanie et au Sénégal
}

\begin{abstract}
EN 1992, LA CONVENTION SUR LA DIVERSITÉ BIOLOGIQUE A GARANTI LES DROITS DES POPULATIONS AUTOCHTONES SUR LA NATURE. DANS LES AIRES MARINES PROTÉGÉES (AMP) OUEST-AFRICAINES, L'APPROCHE CONSERVATOIRE TEND À ENCOURAGER DES FORMES AUTOCHTONES DE RÉGULATION DE L'ACCÈS AUX TERRITOIRES ET AUX RESSOURCES. SI L'AUTOCHTONIE S'EST IMPOSÉE COMME UN RÉFÉRENT GLOBAL, ELLE N'EN SOULÈVE PAS MOINS UN CERTAIN NOMBRE D'AMBIGUÏTÉS QUE RÉVÈLENT ICI LES EXEMPLES DE DEUX AMP EN MAURITANIE ET AU SÉNÉgal. L'AUTOCHTONIE SERAIT-ELLE DAVANTAGE UN PRODUIT DU CAPITALISME GLOBAL CONTEMPORAIN QU'UNE RÉSISTANCE À LA MODERNITÉ MARCHANDE?
\end{abstract}

En Afrique, la colonisation s'est souvent accompagnée de l'exclusion des populations des espaces au plus fort potentiel de biodiversité. Le modèle des parcs nationaux s'est disséminé sur le continent, entérinant une gestion centralisée de la nature par l'État colonial, puis postcolonial. L'État a légitimé la dépossession des populations de leurs droits $d$ 'accès et $d$ 'usage sur les territoires en insistant sur l'altération de la nature causée par les activités anthropiques, tout en occultant les conditions d'exploitation et de «valorisation" de la nature, notamment le mercantilisme qui guidaient l'économie des États en formation. L'accélération de la marchandisation et de la globalisation de l'exploitation de la nature a bouleversé les modes de gestion de la biodiversité. L'acuité des problèmes environnementaux, dont a pris acte le «sommet de la
Terre » de Rio en 1992, a posé la question de l'encadrement du marché global des ressources naturelles, marché où les entreprises transnationales tiennent un rôle majeur. Le renforcement du rôle régulateur des États sur ce marché s'est imposé, mais les collusions fréquentes entre États et groupes marchands ont aussi conduit les participants à mettre l'accent sur l'implication des populations dans la gestion des ressources naturelles ${ }^{1}$.

Ainsi, la Convention sur la diversité biologique (CDB), signée lors du sommet de Rio, a imposé la prise en compte de l'humain dans la gestion de la biodiversité en consacrant l'idée d'un transfert des droits sur la nature aux «populations locales». Si la convention reconnaît aux États une souveraineté en matière de gestion environnementale, l'alinéa J de l'article 8 garantit les droits des populations 
autochtones sur la nature. L'opérationnalisation de cet article a suscité des débats ${ }^{2}$, mais il a permis d'affirmer le principe de la conservation des savoirs naturalistes au moyen de démarches patrimoniales, et de freiner les appétits marchands pour les ressources naturelles. En outre, la CDB, en son article 10, marque la préoccupation des acteurs internationaux pour les savoir-faire qui incarnent des modes de vie traditionnels, considérés comme «durables», et appelle à leur préservation et à leur patrimonialisation ${ }^{3}$. Détentrices de savoir-faire, les communautés autochtones sont instituées acteurs de la gouvernance environnementale, et des droits leur sont reconnus sur les espaces protégés 4 ; leur savoirs naturalistes et leurs usages et formes de régulation sont ainsi consacrées. Malgré le flou connu qui caractérise les notions d'autochtone, de tradition et de local, $\mathrm{l}^{\prime}$ « ancestralité » s'impose dans les textes internationaux comme un critère de gestion durable ${ }^{5}$ : l'antériorité d'installation serait une garantie d'intimité des relations à la terre et de durabilité des techniques de gestion des ressources; c'est elle qui fonderait les droits d'exploitation d'un territoire, définis en droit international et consacrés dans les textes des Nations unies ou du Bureau international du travail relatifs aux peuples autochtones ${ }^{6}$.

C'est à la fin des années 1990, avec l'affirmation de la dimension conservatoire de la gestion environnementale au détriment de sa dimension protectionniste, que la diversité culturelle et l'entretien de la diversité biologique se sont combinés ${ }^{7}$. Les organisations non gouvernementales transnationales jouent un rôle important dans la promotion des régulations "autochtones» de la nature, et elles s'appuient sur des groupes autochtones pour faire prévaloir leur agenda aux dépens des États et des groupes marchands ${ }^{8}$.

Cette mode de la gestion autochtone s'est propagée en Afrique dans un contexte marqué par une fragilisation de la gouvernance étatique et par des processus de démocratisation et de restauration autoritaire propices au surinvestissement politique des questions autochtones ${ }^{9}$. Au cours de cette période, la fascination pour le concept de «société civile» dans le champ du développement ${ }^{10}$, qui a justifié le contournement du gouvernement des États jugé inefficace, a encouragé la «traditionalisation de la gouvernance ${ }^{11_{»}}$. Ce discours et ces arts de faire du développement ont sans doute négligé les manipulations politiques des cadres d'intervention choisis, mais ils n'en ont pas moins stimulé des logiques locales hégémoniques. La démarche patrimoniale qui guide les interventions au niveau des aires protégées s'appuie essentiellement sur la valorisation des identités des populations résidentes, qui est sélective - la dimension politique du classement des savoirs indigènes est souvent occultée ${ }^{12}$-, et qui suscite des revendications autochtones parfois ambiguës.

Les Aires marines protégées (AMP) qui se sont développées dans la foulée du sommet sur le développement durable de Johannesburg en 2002 et du congrès des parcs de Durban en $2003^{13}$, ont, comme d'autres, été construites sur ce modèle, en assimilant les pratiques de conservation à des pratiques culturelles immuables; l'identité des populations et leur histoire sont censées valoriser les espaces à protéger dans le but de préserver la biodiversité. Notons toutefois que les AMP n'ont fait que porter à son terme la logique communautariste depuis longtemps déjà appliquée à la gestion de nombreuses zones 
de pêche ${ }^{14}$. Enfin, l'influence du paradigme des Common Pool Ressources (CPR) dans les années 1990 a placé au premier plan les régulations «autonomes » de l'État et du marché dans l'accès aux ressources environnementales ${ }^{15}$. Les acteurs de la biodiversité qui parcourent les aires protégées ouest-africaines puisent dans ces discours des arguments pour justifier leur action, en effectuant des raccourcis entre gestion commune et gestion autochtone.

L'approche retenue dans le cadre des AMP a ainsi entraîné un renforcement des pouvoirs considérés comme autochtones. Si l'autochtonie s'est imposée comme un référent global, elle n'en soulève pas moins un certain nombre d'ambiguités au niveau local sur le banc d'Arguin (Mauritanie) comme dans le delta du Saloum (Sénégal) étudiés ici ${ }^{16}$. L'insistance sur l'autochtonie ne rompt pas avec la dépolitisation qui caractérise les interventions de développement ${ }^{17}$; elle se traduit par la promotion de modes de gouvernance exacerbant les compétitions et les exclusions dans l'accès aux ressources. Le critère de l'antériorité sur le territoire est en effet inapplicable dans des espaces depuis longtemps extravertis et il est pratiquement en contradiction avec les principes d'une justice distributive, inhérents au domaine environnemental. Cette contradiction n'est-elle qu'un hasard de l'histoire de ces zones? Les revendications autochtones contemporaines ne reflètent-elles pas plutôt des rapports de force plus larges, liés aux conditions d'accès aux droits d'exploitation, dans un contexte de décharge de l'État ${ }^{18}$ ? La globalisation de l'environnement favorise des dynamiques locales qui semblent correspondent davantage à des enclosures (appropriations privatives) ${ }^{19}$ qu'à une gestion durable de la nature. Les démarches autochtonistes seraientelles plus une opportunité pour exclure certains groupes de l'accès aux espaces et aux ressources qu'un moyen efficace de conservation de la nature?

\section{À LA CROISÉE DES INFLUENCES}

Malgré les efforts de «muséification » des cultures matérielles et des connaissances liées à l'utilisation de la nature, les phases de peuplement et l'histoire des savoirs locaux des aires marines protégées restaient mal connues lors de leur création le long du littoral africain. Les dynamiques de peuplement déterminent pourtant les modes d'exploitation des ressources naturelles, et il faut connaître l'histoire des sociétés et des territoires concernés pour identifier les recompositions identitaires et spatiales liées aux AMP. En partant des processus migratoires et des luttes de pouvoir aux périodes anciennes, on peut décrire les dynamiques qui remettent en question l'assimilation des aires marines protégées à des territoires autochtones. Si la délimitation de territoires en fonction de la primauté d'occupation peut sembler simple dans le cas des populations amérindiennes en Amérique du Sud, il en va différemment en Afrique, où les territoires ont connu des vagues migratoires successives et des processus d'assimilation entre communautés de différentes origines ${ }^{20}$.

\section{Le Parc national du banc d'Arguin (PNBA) : la diversité des relations tributaires}

La confluence de groupes berbères, arabes et «noirs» vers le banc d'Arguin a façonné l'identité des habitants d'aujourd'hui, les Imraguen, qui sont une composante de la société maure, l'une de ses strates, marquée 
par des liens de sujétion avec divers groupes tribaux ${ }^{21}$.

Les Imraguen, progressivement spécialisés dans la pêche, sont en fait d'anciens groupes serviles (hrâtîn, artisan/tributaire) ou tributaires des tribus nomades situées aux confins du banc d'Arguin. Ils se sont sédentarisés dans les années 1960. Auparavant, ils ont longtemps été dépendants des tribus régnant à la périphérie de leur zone d'exploitation halieutique, qui détenaient les droits d'exploitation sur les puits, les terres de culture et les zones de pêche. Ce sont les conditions environnementales et climatiques, le manque d'eau douce et leur sujétion à leurs protecteurs pendant la tumultueuse période de la traite 22 qui expliquent la faible fixation et le nomadisme des populations du banc d'Arguin. Les pêcheurs imraguen payaient des redevances en nature aux suzerains tribaux. Le poids de ces tributs, associé à l'insécurité et à la rareté des ressources hydriques, limitait la croissance démographique dans la zone.

Au cours du XVII e siècle, la portion de côte correspondant à l'actuel PNBA a été soumise à l'influence de groupes guerriers trarza, en compétition avec les Awlâd Dlaym, auxquels les Gur', toujours présents dans l'espace qui nous intéresse, sont liés. Les tribus «maraboutiques" des Ahl Bârikalla et des Ahl Buhubbayni, subissant elles-mêmes l'hégémonie des Trarza, nomadisaient également dans la région et y disposaient sans doute déjà de prérogatives foncières ${ }^{23}$. Au XIX ${ }^{\mathrm{e}}$ siècle, quelques familles des Awlâd Busba' commencèrent à s'établir dans la presqu'île d'Iwik et sur l'île de Tidra qui lui fait face. À cette époque, les modes d'exploitation des ressources dépendaient d'un enchevêtrement de différents droits d'usages qui se traduisaient par le versement de tributs aux groupes régnant sur le banc d'Arguin ${ }^{24}$.

La colonisation de la Sénégambie a bouleversé ces rapports hiérarchiques. Sur l'ensemble du territoire, les troupes françaises ont mis longtemps à venir à bout d'une vive résistance. Puis le système colonial s'est employé à organiser le rachat des tributs payés par les dépendants: l'administration a décrété et supervisé le paiement par les Imraguen d'une compensation financière globale à leurs anciens maîtres, censée rendre caduc leur pouvoir ${ }^{25}$. Bien que l'opération n'ait concerné qu'un faible nombre de groupes, elle a permis de légitimer un nouveau pouvoir de régulation dans l'accès aux ressources, qui a partiellement estompé les modes d'accès tributaires.

Au début de la Seconde guerre mondiale, la crise climatique qu'a connue la Mauritanie a contraint une grande partie des populations nomades à se rapprocher des côtes et a contribué à une exploitation beaucoup plus poussée des produits halieutiques ${ }^{26}$. De plus, comme le conflit européen a perturbé l'approvisionnement des colonies, les autorités coloniales ont tenté de développer la production de poisson le long des côtes sénégambiennes. À la fin de la guerre, elles ont organisé le rachat des redevances que les Imraguen payaient encore à l'émir du Trarza, de manière à stimuler les captures. Une entreprise française, la Société industrielle de la grande pêche (SIGP), installée depuis 1919 à Nouadhibou, a participé à cette opération en échange d'un engagement des Imraguen à fournir de la poutargue (œufs de mulet séchés) et du poisson saléséché à prix fixe ${ }^{27}$.

Dans la seconde moitié du XXe siècle, avec la progression du peuplement des côtes, et 
notamment la croissance des agglomérations de Nouadhibou et Nouakchott, les activités, le paysage démographique et les enjeux politico-identitaires de l'espace du PNBA se sont recomposés. L'exploitation des ressources halieutiques s'est encore intensifiée pour servir le marché intérieur et le marché extérieur, de plus en plus important ${ }^{28}$. Les techniques ont évolué par des apports exogènes et des innovations endogènes, et le nombre d'embarcations du banc d'Arguin a fortement crû. L'émancipation des populations du banc au cours de la colonisation s'est accompagnée de flux migratoires, qui rendent l'identité imraguen difficile à circonscrire. Les dynamiques de peuplement et les changements qu'elles ont entraînés ont compliqué les opérations de recensement des populations et des pratiques effectuées depuis la création du parc par l'État mauritanien, en 1975.

Bien que la période coloniale ait quelque peu figé l'identité imraguen, après la déstructuration des modes d'accès aux territoires et des types d'exploitation tributaires, l'appartenance à cette communauté est aujourd'hui devenue un enjeu de pouvoir révélé par les stratégies de compétition. Avec la création du PNBA et l'amorce d'une «muséification » des populations (imraguen), enrôlées dans la conservation du milieu naturel, les habitants du parc ont en quelque sorte été sommés de s'identifier au(x) projet(s) dont ils étaient les «bénéficiaires ». L'identité écologique qu'on leur proposait d'endosser les a encouragés à se rapporter au cadre tribal d'appropriation et de contrôle de l'espace. Les autorités administratives et les intervenants extérieurs (les porteurs de «projets »), pris au piège de la conservation qu'ils invoquaient, ont ainsi aidé, consciemment ou non, à la réinvention de ces identités tribales, les groupes étant parfois désignés, de manière plus politiquement correcte, sous le nom de «coopérative» ou de « comité local» 29 .

Les enjeux de pouvoir liés aux velléités d'appropriation des ressources ou du potentiel de valorisation de l'espace du PNBA ont réactualisé les relations tributaires, malgré l'ouverture croissante de l'aire du parc à de multiples acteurs. Les migrations et l'essor du marché au cours du XXe siècle se sont intégrés à ce cadre hiérarchique qui continue d'agir sur les modes d'accès aux ressources. La manipulation du capital économique et politique par les caciques des tribus, qui voyaient dans la création du parc des opportunités d'accumulation, a réinscrit les populations de pêcheurs imraguen dans les dispositifs de pouvoir anciens.

\section{La Réserve de biosphère du delta du Saloum (RBDS) : des conquêtes aux migrations de travail}

L'histoire a aussi beaucoup pesé sur les pratiques d'exploitation des ressources naturelles dans la Réserve de biosphère ${ }^{30}$ du delta du Saloum, créée en 1976, avec une diversité d'influences qui ont façonné des savoirs, des usages et des identités plurielles. Le delta du Saloum se partage en deux zones, les îles du Gandoul au nord et les îles du Niombato au sud, qui sont aujourd'hui peuplées respectivement par des Sérères Niominka et des Socé, appartenant au groupe mandingue. Cette démarcation recoupe en partie les zones d'influence des entités historiques qui ont exercé un pouvoir sur le delta, le royaume sérère du Siin au nord, et le royaume mandingue du Gabu au sud ${ }^{31}$. Ces royaumes ont contrôlé les parties continentales du Saloum 
étroitement, tandis que sur les îles, leur pouvoir était plus lâche.

Au cours des phases de conquête et lors de la traite atlantique, la pression exercée par les deux royaumes a poussé des populations nouvelles vers les îles du Saloum. Ces populations se sont progressivement incorporées aux groupes autochtones, ce qui explique que les insulaires d'aujourd'hui (mais plus rarement leurs chefferies) se revendiquent souvent des deux origines. Dans les îles du nord, les populations sérères ont accueilli peu de migrations mandingues, dont les seules traces se retrouvent dans certains rituels. En revanche, le Niombato, au sud, a connu un afflux migratoire à la fin du XIXe siècle, suite à la conquête musulmane, qui a fait refluer une partie de la population des îles vers le sud-est. Des populations sérères migrantes se sont alors assimilées aux Mandingues au point d'abandonner leur langue d'origine. Dans le delta du Saloum, il semble que la distinction Sérère/Socé date de peu avant la conquête, et que cette séparation soit plutôt d'ordre politique, le Gandoul se rattachant au Siin et le Niombato plutôt au Gabu. L'organisation socio-familiale semble néanmoins davantage marquée par l'influence sérère ${ }^{32}$.

Le commerce entre les îles et leur environnement régional s'est développé très tôt, ainsi qu'en atteste l'essor des réseaux commerciaux baks et bainouks du $\mathrm{x}^{\mathrm{e}}$ au $\mathrm{XV}^{\mathrm{e}}$ siècle. Il s'est ensuite prolongé le long de la côte, vers les comptoirs européens de Joal, de Palmarin et du fleuve Gambie ${ }^{33}$. Les insulaires échangeaient alors leur production vivrière contre des produits de traite ${ }^{34}$. Si ces échanges locaux sont les plus dynamiques, la zone du Saloum participe d'un commerce plus étendu. La greffe de la traite atlantique sur la traite saharienne a intensifié l'exploitation des produits de la mer et a poussé à une certaine spécialisation des insulaires, en particulier dans le Gandoul, où les terres salées se prêtent moins à l'agriculture que celles du Niombato.

La période coloniale a entériné cette dichotomie, en insérant le Gandoul dans une économie maritime basée sur le commerce de produits vivriers et sur la contrebande avec la Gambie anglaise et la Guinée portugaise ${ }^{35}$. Au début du Xxe siècle, ce commerce a bénéficié d'une innovation technologique, la pirogue à quille, construite avec de gros troncs d'arbre. Après la Seconde guerre mondiale, la spécialisation halieutique des Niominka s'est affirmée, les activités de pêche s'intensifiant avec la pratique de la navigation en mer $^{36}$. En migrant vers les villes côtières et surtout vers Dakar, les Niominka ont intégré les circuits de commercialisation des produits de la pêche. Leur connexion au milieu du mareyage, puis de la pêche industrielle thonière, a contribué à capitaliser la pêche piroguière menée depuis le delta du Saloum. Par la mer, les Niominka ont renforcé leur présence à l'intérieur et aux confins du delta: ils se sont implantés pour pêcher dans les lieux autrefois fréquentés de manière saisonnière pour la culture du riz. $\mathrm{Si}$ les anciens campements des îles du Gandoul ont été privilégiés, ceux du Niombato sont également fréquentés. Les populations du Niombato ont profité de leur côté d'un commerce de contrebande plus localisé avec la Gambie anglaise, mais elles sont restées tournées vers l'agriculture.

L'immigration vers le delta a crû fortement dans la période coloniale. Au début du XXe siècle, les migrants ont afflué vers la partie continentale du delta, qui accueille plusieurs points de traite. Par ailleurs, jusque dans les 
années 1970, la culture de l'arachide a attiré vers la zone continentale des Wolofs, ainsi que des Bambara en provenance du Soudan. À partir des années 1970, des Toucouleurs se sont installés dans les îles pour pêcher la crevette dans le delta. Cette économie crevettière a drainé, de façon saisonnière, des migrants venant des États riverains du Sénégal (Mali, Gambie, Guinée-Bissau). À la fin des années 1970, l'implantation d'une usine de farine de poisson dans le campement de Djiffer a renforcé l'attractivité de la zone, et des Lebou, de langue wolof, originaires de la Petite Côte et de Saint-Louis, s'y sont implantés pour pêcher dans les chenaux du delta et sur la façade maritime.

Tout au long du xxe siècle, le brassage des populations du delta du Saloum s'est accentué, même si les îles sont demeurées à forte dominante socé et niominka. La migration saisonnière liée à l'exploitation des ressources halieutiques est demeurée très importante, y compris dans les parties insulaires du delta du Saloum. Mais ce processus, qui concerne surtout les Niominka, s'est accompagné d'une dissémination des populations insulaires des villages vers les campements de pêche, dont certains sont devenus des lieux d'habitation permanents.

Sur le banc d'Arguin comme dans le delta du Saloum, traites atlantique et saharienne ont redistribué les populations; la colonisation a stimulé une économie maritime qui a tiré profit de la disparité des systèmes coloniaux et ouvert des échanges. Ces processus ont installé dans les zones littorales des populations aux origines très variées. Cette diversité interroge le choix de l'autochtonie comme critère de la régulation de l'accès aux espaces et aux ressources. L'examen des technologies de capture ou de transformation des produits montre d'ailleurs qu'elles ont incorporé des pratiques venues des peuplements allochtones et de l'ouverture aux échanges extérieurs. Peut-on dans ces conditions, préjuger de l'efficacité d'institutions autochtones pour réguler l'accès aux ressources naturelles?

Le banc d'Arguin et le delta du Saloum se sont intégrés de manière différente au marché. Les communautés du Saloum (et les Niominka plus encore que les Socé) se sont très tôt engagées dans l'économie maritime. Les pêcheurs imraguen du banc d'Arguin en revanche, en raison de leur statut de dépendants, sont devenus une main-d'œuvre pour des armateurs mieux placés dans la hiérarchie économique et politique. Leur insertion dans le capitalisme mondialisé de la production halieutique, plus récente qu'au Sénégal, malgré l'incorporation de techniques exogènes, les a cantonnés dans une position plus marginale. Comme nous allons le voir, cette distinction s'est traduite dans les faits par une différence dans le statut accordé à l'autochtonie dans la gestion des AMP. Nous nous interrogerons également sur l'enjeu de l'autochtonie et la manière dont il reconfigure les pouvoirs locaux dans ces zones de biodiversité.

\section{GouVERNANCE ET AUTOCHTONIE}

Malgré la difficulté que pose la notion d'autochtonie dans des espaces aussi travaillés par l'histoire et la faible connaissance qu'ont les populations des textes relatifs aux droits des peuples autochtones, l'adhésion des groupes de pression internationaux à une gestion de la biodiversité basée sur l'autochtonie conduit à cibler les interventions en direction quasi exclusive des primo résidents. Dans un contexte où les identités sont plurielles, et où 
les modes de valorisation des ressources sont différents, l'engagement des communautés locales dans un processus de conservation qu'elles n'ont guère contribué à définir ne sert-il pas de paravent à des modes d'exploitation peu en accord avec les objectifs du développement durable? Dans les AMP ouestafricaines, la promotion de l'autochtonie, modulée selon les niveaux de délégation des droits d'accès aux espaces et aux ressources naturelles, soulève en tout cas des questions.

\section{LE BANC D'ARGUIN : LES IMRAGUEN AUX PRISES AVEC LES LIENS TRIBUTAIRES}

C'est sans doute dans le banc d'Arguin que l'autochtonie intervient le plus directement. L'assimilation des populations résidentes à l'identité imraguen s'est concrétisée à partir de la période coloniale. Par la suite, les représentations des scientifiques, de l'État et d'autres acteurs ont charrié une vision tronquée de la communauté résidente - une communauté de pêcheurs vivant en osmose avec la nature, pratiquant la pêche à pied grâce à l'aide des dauphins. Cette vision idyllique de «l'homme de la nature ${ }^{37} »$ a focalisé les interventions sur les Imraguen, tout en reconnaissant le bien-fondé des droits d'accès tribaux aux ressources naturelles. Comme l'espace du banc d'Arguin a été assimilé à un type de pêche (le mulet jaune), et à une certaine identité (la communauté imraguen qui pratiquait cette pêche), la question du recensement et de la catégorisation de cette communauté a émergé dans le cadre de la gestion du PNBA.

Le statut d'aire protégée a révélé l'acuité du problème de l'autochtonie, dans la mesure où la crise écologique des trente dernières années a attiré vers la côte des populations nouvelles, et a suscité la mise en exploitation de nouvelles espèces (requins, raies, courbines) et l'emploi de techniques nouvelles (nouveaux types de filets et essor de la pêche motorisée). Les Imraguen sont alors entrés au service d'entrepreneurs issus de leur hiérarchie tribale, au statut plus élevé et qui avaient les moyens d'investir dans le secteur. Le changement de statut lié à la création du parc a aussi aiguisé les appétits d'investisseurs nationaux. Mais l'intensification de l'exploitation a conduit les autorités à réglementer davantage les activités de capture, au profit des populations autochtones: depuis 1998, la pêche motorisée est interdite dans le territoire du PNBA et les pêcheurs n'utilisent plus que des lanches, des embarcations à voile d'origine espagnole; en outre, les armateurs qui opèrent dans le parc sont tenus de recruter pour chaque embarcation parmi les résidents leur capitaine et au moins deux membres d'équipage (sur cinq en moyenne).

Pour veiller à une exploitation durable, des réunions de concertation ont lieu chaque année entre des représentants des pêcheurs et l'administration du parc. Elles engagent essentiellement les résidents (donc les Imraguen et les représentants des rangs plus élevés dans leur tribu, les bizân), par le biais des coopératives, excluant du même coup les armateurs allochtones, dont les embarcations motorisées opèrent à la périphérie de l'aire protégée. Ces réunions donnent parfois lieu à d'âpres négociations. Il ne s'agit pas tant de définir des droits d'accès, dont la régulation reste du ressort de l'organisation tribale ou pour le moins est inféodée au critère de l'identité et de la résidence, mais plutôt d'établir des restrictions sur les types d'équipement, afin de limiter l'effort de pêche. Cet aménagement des pêches 
dans le cadre du PNBA atténue en partie la pression des armateurs situés en dehors du parc.

La négociation conduit aussi à des arbitrages entre les différents intérêts en compétition, même si les hiérarchies tribales influencent les prises de décision. Ainsi les affrontements n'opposent pas tant les tribus entre elles que différents segments au sein d'une même tribu, notamment à propos du contrôle de la force de travail des catégories assujetties (en l'occurrence les Imraguen, dont la plupart sont, comme on l'a vu, des hrâtîn). Les hiérarchies tribales s'imposent aux dépens de leurs dépendants imraguen, les propriétaires les plus importants de lanches étant généralement des mareyeurs et des transporteurs de statut social élevé (bizân). C'est seulement avec l'émancipation graduelle des groupes assujettis, amorcée à l'époque coloniale, et avec l'insertion, plus tardive, du monde imraguen dans l'entreprenariat de pêche, que l'accès aux ressources halieutiques dans l'aire $d u$ PNBA a progressivement fait apparaître une divergence entre «investisseurs » et intermédiaires des marchés régionaux (Nouakchott et Nouadhibou) d'un côté et pêcheurs "de base» locaux de l'autre - certaines familles de hrâtîn, à l'instar de celles du village d'Agadir, ont acquis de longue date leur autonomie vis-àvis des bizân.

Si les patrons de statut dit «élevé » recourent systématiquement à des employés, la situation est plus difficile à démêler pour ceux qui appartiennent à la catégorie des « dépendants » (hrâtîn). Une fraction non négligeable des hrâtîn qui se déclarent propriétaires travaille probablement, selon des contrats aux formes variées, pour le compte d'un "patron ». Et l'on remarque dans presque tous les villages, autour de clans locaux influents de même extraction, un début d'accumulation. Une partie de l'exploitation de la force de travail, qui se réalisait jadis au travers des structures hiérarchiques traditionnelles, tend aujourd'hui à s'opérer au sein même de la communauté imraguen, entre hrâtîn, où se recrute désormais une partie des armateurs mais aussi leurs employés, cadets de famille ou simples auxiliaires appointés ${ }^{38}$.

Pourtant les cloisonnements anciens sont loin d'avoir disparu, et les interventions extérieures visant à maintenir ou restaurer les équilibres «naturels» dans la zone du parc sont à cet égard ambiguës. Les tribus, cadre traditionnel de légitimation de l'accès aux ressources et marqueur essentiel des regroupements démographiques sur le terrain, peuvent difficilement être contournées. Même si les contacts et les décisions ne se font pas ouvertement en leur nom, les figures majeures, les personnes d'influence dans le parc, Imraguen ou non, qui s'engagent et décident au nom des autres, et qui ne résident d'ailleurs pas nécessairement sur place, inscrivent leur autorité dans le cadre tribal. Ce sont aussi elles qui tirent le plus avantage des moyens et des ressources drainées par les interventions extérieures, même si les mécanismes de redistribution viennent souvent tempérer la prédation des intermédiaires.

Le statut d'Imraguen conférant un certain nombre de droits sur les ressources naturelles, l'appartenance à cette communauté et sa représentation sont devenues un réel enjeu politique. Les tribus établies en dehors du parc, notamment celles qui disposaient de droits anciens, affirment aujourd'hui leurs droits d'accès à cet espace; elles remobilisent des liens hiérarchiques anciens avec les populations 
imraguen, en employant leur accès privilégié à l'appareil d'État. Les stratégies en ce sens sont diverses, de l'armement de bateaux en proche périphérie du parc à des revendications territoriales strictes, voire à des investissements touristiques dans le parc - pour accéder aux dividendes du statut d'AMP et profiter d'une clientèle touristique captive, astreinte par la réglementation à user des équipements tribaux payants installés sur place. En outre, les migrations de travail dans le parc tendent à s'incorporer au système tribal: les arrivants s'inscrivent dans une démarche d'autochtonisation. C'est ainsi que des migrants de statut modeste, parfois issus de tribus lointaines, s'intègrent aux Imraguen par le mariage. La multiplication de ce type de mariages et la captation ainsi opérées des gendres allochtones pourrait bien remettre en cause les équilibres existants.

La question de l'identité revêt une acuité particulière quand il s'agit de proroger ou d'acquérir un statut d'interlocuteur reconnu par les institutions du parc. Les modes de gouvernance qui mettent l'accent sur l'autochtonie exacerbent les compétitions internes à la communauté imraguen pour la représentation des populations face aux autorités et aux diverses instances intervenant dans le parc. Les intermédiaires hiérarchiques agréés, repérés selon le critère de l'autochtonie, sont de facto souvent les premiers bénéficiaires des interventions extérieures. Il en découle une réactivation des relations de patronage, qui a pour corollaire une représentation clientéliste des populations du parc, les hiérarchies tribales jouissant de réseaux d'accès à l'État, au détriment des Imraguen dépendants. Malgré leurs attentes divergentes, toutes les parties coopèrent dans le cadre des projets initiés au sein du PNBA.
Le contexte contemporain est marqué par une recomposition des modes de territorialité tributaire, même si les bénéfices attendus des interventions extérieures en termes de ressources économiques et/ou de «représentativité» (prestige, honneur, pouvoir...) suscitent quelquefois des scissions ou de nouveaux établissements. Les fondateurs de Tissot ont sans doute abandonné leur village pour se soustraire à l'autorité de leurs anciens " patrons » tribaux et affirmer leur autonomie. Cette stratégie répond sûrement aussi à une volonté de «branchement direct» sur le réseau institutionnel du parc et sur ses bénéfices matériels et symboliques supposés. Néanmoins, si certains pêcheurs imraguen s'émancipent, au point que les inégalités se développent au sein de cette communauté, la gouvernance basée sur l'autochtonie les contraint à s'inscrire dans des hiérarchies tribales qui peuvent leur être défavorables, leurs anciens suzerains réaffirmant leurs droits. Au-delà de la mise au premier plan de l'autochtonie se jouent des modalités de contrôle de la force de travail, $d$ 'une part entre les Imraguen, et d'autre part entre les Imraguen et les caciques des tribus auxquelles ils appartiennent.

\section{LE DELTA DU SALOUM :}

\section{LES SURENCHÈRES AUTOCHTONES}

La question de l'autochtonie dans le delta du Saloum se joue d'une manière assez différente, car ce n'est pas une norme reconnue dans les règlements définissant l'accès aux ressources de l'AMP. Néanmoins, l'autochtonie n'est pas absente de la gouvernance de la réserve, dans la mesure où elle influence des interventions en marge de celles menées par l'État, qui ciblent les populations tenues pour primo résidentes. Les ONG internationales 
ou nationales ont privilégié une approche mobilisant l'autochtonie pour mieux réguler l'accès aux ressources naturelles dans un contexte de retrait de l'État, alors que les collectivités locales, faute de ressources institutionnelles et financières, ne parviennent guère à s'affirmer. La démarche s'est imposée avec la défiance suscitée par les normes publiques de gestion des ressources issues de la loi sur le domaine national. Cette loi, élaborée en 1962, était destinée à élargir l'accès à la terre pour le paysannat, en faisant de tout exploitant le détenteur d'un droit d'usage vis-à-vis de l'État («la terre appartient à celui qui l'exploite»). Elle visait à déposséder les chefferies de leur pouvoir au profit de l'État, devenu le garant de l'accès du plus grand nombre aux ressources naturelles. Sans saper le pouvoir des élites locales (néo)traditionnelles, elle a permis d'étendre un pouvoir clientéliste ${ }^{39}$. Son extension au domaine maritime a fait des territoires de pêche des zones d'exploitation pour tout pêcheur artisan («la mer appartient à tous » selon l'adage), alors qu'auparavant, les territoires de pêche pouvaient être régis par des instances de régulation locale.

Dans la mise en œuvre des restrictions à l'exploitation au sein de l'AMP, les interventions des ONG ont visé à contourner la norme étatique, considérée comme favorisant les situations de libre accès à la ressource halieutique, en encourageant les modes de contrôle autochtones - les institutions villageoises, se basant sur l'autorité des premiers occupants des villages, étaient réputées plus efficaces. Des comités de plage, composés de pêcheurs autochtones, ont été créés début 2000 sous l'impulsion de l'Union internationale pour la conservation de la nature (UICN) qui a opté pour un appui aux organisations villageoises du delta. Sous la houlette des chefferies villageoises, ces comités ont accueilli des représentants des lignées reconnues comme originaires du village et organisées en quartiers. Ils ont instauré une série de restrictions, dans l'espace, dans le temps, sur des techniques ou sur la taille des espèces capturées. La fermeture temporaire des passes (bolons) autrefois dédiées à la pêche des seuls résidents a alors été retenue pour sa conformité à la gestion autochtone passée, mais elle s'applique désormais à l'ensemble des pêcheurs.

La concession territoriale, qui repose sur le principe qu'à une communauté correspond un territoire d'exploitation, a ainsi été expérimentée. Or, elle bute sur la réalité des formes de territorialisation des activités dans et en dehors de la RBDS, qui procèdent de dynamiques d'immigration et d'émigration fortes et anciennes. Le phénomène migratoire rend difficile une régulation de la pêche à partir des seuls comités de plage, qui sont organisés sur une base villageoise sans moyens de contrôle sur un vaste «territoire de parcours ${ }^{40}$. La colonisation halieutique du delta du Saloum est surtout le fait de la pêche autochtone niominka. Mais comme on l'a vu plus haut, elle est aussi entretenue par la pêche allochtone lebou, en provenance des régions situées au nord du Saloum et du pays. Ce nouveau mode de régulation porté par les ONG bute également sur le cadre juridique sénégalais: les dispositions de la loi sur le domaine national n'ont pas été aménagées, et l'accès au territoire est donc ouvert à tous ceux qui l'exploitent.

L'action des ONG a stimulé les revendications autochtones et les compétitions sur l'espace. Ainsi, la création, sous l'égide d'une ONG nationale, de la réserve du Bambouk 
(englobant le campement bambouk ainsi que tout le bolon) a favorisé la gestion autochtone $\mathrm{d}$ 'une zone où les usages étaient pourtant partagés par différentes communautés. La prohibition des activités de pêche et de cueillette des coquillages s'est faite au seul profit du village socé de Toubacouta, cheflieu de la Communauté rurale, avec la création d'un projet écotouristique géré par les villageois. En revanche, les ressortissants niominka du village de Bassoul, qui ramassaient des coquillages dans la zone, en revendiquent toujours l'accès, entre autres au nom de l'ancienneté de leur installation dans la zone - un certain nombre de Bassoulois sont nés dans le campement précité. La formation de cette réserve par l'ONG n'ayant pas tenu compte de l'ancienne « relation de tutorat ${ }^{41}$ » entre Socé et Niominka - avec l'autorisation des habitants socé de Toubacouta, les Niominka avaient, voici plusieurs décennies, défriché des terres situées dans la réserve pour y cultiver -, la légitimation de l'accès s'est muée en une surenchère juridique, le «droit de hache» mis en avant par les Niominka (qui découle de leur rôle dans le défrichage de la zone) s'opposant au droit des premiers occupants socé.

La montée en puissance des revendications autochtones trouve une illustration dans la stigmatisation de la pêche lebou, que les comités de plage définissent comme destructrice pour l'environnement. La forte compétition entre les pêcheries motorisées autochtone et allochtone, pêche au filet fixe des Lebou et pêche au filet dérivant des Niominka, s'exacerbe. Les comités de plage dénoncent ainsi l'utilisation de filets en nylon par les Lebou, leur usage des branches de palétuviers dans les casiers à seiche ou l'étroitesse des mailles des filets. A contrario, ils présentent leurs méthodes de capture comme durables, les mailles des filets dérivants étant plus larges. Les comités revendiquent un amendement de la loi du domaine national en leur faveur, et utilisent la dynamique d'autochtonie pour tenter de rétablir un contrôle sur les populations migrantes, en particulier sur les saisonniers ${ }^{42}$.

Les conflits s'aiguisent aussi au sein même des communautés de pêcheurs autochtones. La prédilection pour des régulations à l'échelle villageoise et la mobilisation de la chefferie n'ont pas été sans effets sur la revendication autochtone dans les villages du delta du Saloum. Dans le Gandoul notamment, des conflits sont apparus entre les représentants de la chefferie villageoise et les descendants des premiers occupants dans les villages où ces positions n'étaient pas détenues par un même lignage. Dans certains villages, l'alkali, maître des terres en tant que descendant direct des premiers occupants, s'est opposé aux régulations prônées par le comité de plage; ailleurs, ce sont les régulations à son initiative qui ont été repoussées par la chefferie villageoise et le comité de plage. Cette concurrence entre institutions autochtones s'explique par une compétition croissante pour la captation de la rente $d u$ «développement durable», au moment où l'environnement devient une assise des légitimités politiques locales.

Dans les deux espaces considérés, bien qu'aucun processus de concession communautaire n'ait été mis en œuvre directement par les États mauritanien et sénégalais ${ }^{43}$, le paradigme de l'autochtonie guide une série d'actions d'aménagement ou de gestion. La définition même du mode légitime d'accès 
aux ressources fait l'objet d'une compétition au niveau local, qui revêt des formes diverses et peut entraîner des conflits entre les communautés exploitant les ressources des AMP ou au sein même de chacune d'entre elles.

$C^{\prime}$ est sur le banc d'Arguin que le rôle accordé à l'autochtonie paraît le plus important. La consolidation d'une identité imraguen amorcée dès la période coloniale se poursuit aujourd'hui. Alors que les migrants affluent sur le littoral où les attirent des opportunités nouvelles de valorisation des ressources halieutiques, le statut autrefois marginal d'Imraguen confère des avantages dans l'accès aux ressources naturelles; cette identité s'en trouve valorisée et devient un enjeu politique et économique central. Des revendications autochtones, fondées sur des légitimités historiques, sont ainsi avancées par les hiérarchies tribales anciennes pour réactiver des liens de sujétion et de clientélisme. Dans la Réserve de biosphère du delta du Saloum, face à la surexploitation des ressources halieutiques et grâce à la promotion de l'autochtonie par les $\mathrm{ONG}$, les originaires du Saloum ont invoqué une exclusivité sur l'accès aux espaces pour recouvrer un contrôle sur les populations migrantes en tentant de transformer leurs droits d'usage en droits territoriaux.

En définitive, dans les deux aires, les revendications autochtones aiguisent la compétition plus qu'elles ne stabilisent l'accès aux ressources ou qu'elles ne limitent les pressions anthropiques. Mise en œuvre à des degrés divers, selon les trajectoires étatiques mauritanienne et sénégalaise, la gestion autochtone encourage des stratégies d'exclusion entre communautés et en leur sein même. Depuis l'indépendance, l'État sénégalais a cherché à saper les modes de régulation autochtones de l'accès aux ressources naturelles, notamment par l'intermédiaire de la loi sur le domaine national, et les lois de décentralisation n'y ont rien changé, en dépit des demandes des ONG nationales pour adapter le cadre réglementaire. Les politiques de décentralisation sont plus récentes en Mauritanie, ce qui peut expliquer le maintien de structures tribales dans la gestion des ressources au niveau local. L'absence d'institutions intermédiaires dans la représentation des populations explique la force de l'expression communautaire des intérêts locaux, et le rôle des structures tribales dans la régulation de l'accès aux ressources. Néanmoins, dans les deux cas étudiés, la décharge opérée par des États en situation d'ajustement et la globalisation des enjeux environnementaux sont à l'origine d'un même encouragement aux régulations autochtones $\mathrm{d}$ 'accès aux ressources.

Le poids des groupes de pression internationaux est indéniable et explique certainement la tendance à promouvoir l'autochtonie quelles que soient les situations politiques. Dans les AMP ouest-africaines, les premiers arrivants tentent de contrôler les ressources par différentes stratégies auxquelles les interventions des groupes de pression internationaux offrent un cadre approprié. Cette problématique stimule l'ethnicisation de revendications qui sont guidées moins par une démarche de conservation des milieux naturels que par des volontés d'appropriation territoriale ${ }^{44}$. D'une manière générale, la vogue de l'autochtonie a dissimulé ses propres ambiguités et ses limites, l'application concrète de ses principes dans les modes de gouvernance étant rarement questionnée. Les groupes locaux ont ainsi vu leur pouvoir 
se renforcer, pour le meilleur - quand ils ont pu rétablir leurs droits face aux États ou aux groupes marchands internationaux -, comme pour le pire - lorsque l' « invention de la tradition ${ }^{45}$ » qu'ils ont mise en œuvre, surtout destinée à exclure des groupes des territoires d'exploitation des ressources patrimoniales, est susceptible de revêtir des formes violentes ${ }^{46}$.

La Convention sur la diversité biologique s'est donc traduite dans les aires considérées par la forte affirmation du caractère autochtone de la régulation de l'accès aux ressources. Comme la définition de l'autochtonie néglige la complexité des identités et de l'histoire des espaces, elle est lourde de contradictions. L'insistance sur l'antériorité reflète sans doute un engouement trop prononcé pour la gestion des «communaux», qui repose sur le principe qu'à un espace correspond un groupe social usant d'une ressource. Or ce type de situation correspond mal aux contextes africains ${ }^{47}$, où l'exploitation des ressources naturelles est souvent partagée entre des groupes différents et influencée par des dynamiques migratoires. Si l'approche par les communaux a pu paraître séduisante, c'est sans doute parce qu' elle postulait la capacité des communautés à réguler l'accès aux ressources en dehors d'institutions englobantes telles que l'État. La conception de la démocratie libérale qui prône l'autonomisation de la société par rapport à l'État explique la popularité du modèle de gouvernance autochtone auprès des promoteurs des AMP.

Mais la question politique du partage des ressources persiste. Les groupes de pression sont aujourd'hui plus enclins à définir des critères de distinction des usagers qu'à maîtriser les processus de différenciation issus de l'exploitation marchande. Dans cette approche autochtoniste, le poids des normes tend à occulter la dimension politique de leur production. Cette conception favorise ceux qui, au sein des communautés locales, sont les mieux à même d'influencer la production des normes à travers la manipulation de réseaux de pouvoir économiques et politiques. Le paradoxe de l'autochtonie est que son érection au niveau global en instrument de résistance au marché produit localement des rapports de pouvoir sur lesquels peuvent aisément se greffer des stratégies d'appropriation et d'exclusion (enclosure), préalables à une valorisation marchande des espaces naturels. Ainsi, comme le suggèrent Geschiere et Nyamnjoh ${ }^{48}$, 1'autochtonie est peut-être davantage un produit du capitalisme global contemporain qu'une résistance à la modernité marchande...

\section{Tarik Dahou}

IRD, UR 200 «Patrimoines locaux et stratégies »

EHESS, Centre d'études africaines

(CEAf)

\section{Abdel Wedoud Ould Cheikh}

Université de Metz

1. Nous remercions Vincent Foucher et Marie-Christine Cormier-Salem pour leur lecture critique d'une précédente version de ce texte.

2. L. Bérard et al., «Savoirs et savoirs-faire naturalistes locaux. L'originalité française», VertigO, vol. 6, n 1, 2005, sur $<$ www.vertigo.uqam.ca $>$.

3. B. Roussel, «Savoirs locaux et conservation de la biodiversité: renforcer la représentation des communautés ", Mouvements, $\mathrm{n}^{\circ} 41,2005$, p. 82-88.

4. M. Hufty, «La gouvernance internationale de la biodiversité», Études internationales, vol. 32, n 1, 2001, p. 5-29. 5. B. Roussel, «Représenter la diversité», Courrier de la Planète, $\mathrm{n}^{\circ} 75,2005$, p. 52-54.

6. A. Tiouka et P. Karpe, «Droits des peuples autochtones 


\section{Politique africaine}

187 L'autochtonie dans les Aires marines protégées

à la terre et au patrimoine", Journal d'agriculture traditionnelle et de botanique appliquée, vol. 40, n 1-2, 1998, p. 611-634; F. Braem, «Les peuples indigènes en quête de partenaires", in S. Bahuchet (dir.), Les Peuples des forêts tropicales aujourd'hui. Vol. 2 : une approche thématique, Bruxelles, Université libre de Bruxelles (ULB), Programme Avenir des peuples des forêts tropicales, 2000, p. 566-572.

7. M.-C. Cormier-Salem et B. Roussel, «Patrimoines et savoirs naturalistes locaux», in J.-Y. Martin (dir.), Développement durable? Doctrines, pratiques, évaluations, Paris, IRD, 2002, p. 126-142 ; D. Dumoulin, "Légitimités croisées", Courrier de la Planète, $\mathrm{n}^{\circ} 75,2005$, p. 24-29.

8. Voir D. Dumoulin et E. Rodary, «Les ONG au centre du secteur mondial de la conservation de la biodiversité", in C. Aubertin (dir.), Représenter la nature? ONG et biodiversité, Paris, IRD, 2005, p. 59-98; M. Roué, «NGOs, indigenous peoples and local knowledge: issues of power in the arena of biodiversity ", International Social Sciences Journal, vol. 55, $\mathrm{n}^{\circ} 178,2003$, p. 533-537.

9. J.-F. Bayart, P. Geschiere et F. Nyamnjoh, «Autochtonie, démocratie et citoyenneté en Afrique ", Critique internationale, $\mathrm{n}^{\circ} 10$, 2001, p. 177-194; J.-P. Chauveau, «Question foncière et construction nationale en Côte d'Ivoire: les enjeux silencieux d'un coup d'État ", Politique africaine, $n^{\circ} 78$, juin 2000, p. 94-125; J.-P. Dozon, «La Côte d'Ivoire entre démocratie, nationalisme et ethnonationalisme,", Politique africaine, $\mathrm{n}^{\circ} 78$, juin 2000, p. 45-62.

10. J. et J. Comaroff (ed.), Civil Society and the Political Imagination in Africa, Chicago, University of Chicago Press, 1999.

11. L'expression est empruntée à V. Foucher, «"Tradition africaine" et résolution des conflits. Un exemple sénégalais", Politix, n 80, 2007, p. 59-80.

12. A. Agrawal, "Classification des savoirs autochtones, la dimension politique", Revue internationale des sciences sociales, $\mathrm{n}^{\circ} 173,2002$, p. 325-336.

13. Ces sommets ont recommandé la création avant 2012 d'un réseau mondial d'aires marines protégées afin de protéger la diversité biologique marine et les processus écosystémiques.

14. J.-P. Chauveau, E. Jul-Larsen et C. Chaboud (dir.), Les Pêches piroguières en Afrique de l'Ouest. Dynamiques institutionnelles: pouvoirs, mobilités, marchés, Paris, Karthala, 2000.

15. Par opposition à l'idée, défendue par Hardin, selon laquelle l'absence de droits de propriété privée entraîne libre accès aux ressources, qui conduit à l'épuisement des ressources non renouvelables, le courant des CPR analyse comment les ressources non renouvelables sont gérées communautairement par des groupes d'exploitants, selon des règles $\mathrm{d}^{\prime}$ accès et $\mathrm{d}$ 'utilisation susceptibles de les préserver. Voir G. Hardin, «The tragedy of the commons", Science, vol. 162, n 3859, 1968, p. 1243-1248; E. Ostrom, Governing the Commons. The Evolution of Institutions for
Collective Action, Cambridge, Cambridge University Press, 1990 ; E. Ostrom et al. (eds), The Drama of the Commons, Washington, National Academy Press, 2002.

16. Pour un repérage géographique, on se reportera aux cartes annexées au present article.

17. Voir J. Ferguson, The Anti-Politics Machine: «Development ", Depoliticization and Bureaucratic Power in Lesotho, Cambridge, Cambridge University Press, 1990 ; J.-P. Olivier de Sardan, Anthropologie et développement: essai en socioanthropologie du changement social, Paris, Karthala, 1995; E. Garland, «Developing bushmen: building civil(ized) society in the Kalahari and beyond", in J. et J. Comaroff (eds), Civil Society..., op. cit., p. 72-103. Pour une écologie politique des enjeux environnementaux, voir K. S. Zimmerer et T. Basset (eds), Political Ecology. An Integrative Approach to Geography and Environment-Development Studies, Londres, The Guilford Press, 2003; et C. Zerner, People, Plants and Justice, the Politics of Nature Conservation, New York, Columbia University Press, 2000.

18. B. Hibou, "La décharge, nouvel interventionnisme», Politique africaine, $\mathrm{n}^{\circ} 73$, mars 1999, p. 6-15.

19. K. Polanyi, La grande transformation. Aux origines politiques et économiques de notre temps, Paris, Gallimard, 1983.

20. Voir S. Bahuchet (dir.), Les Peuples des forêts tropicales aujourd'hui. Vol. 1-4, Bruxelles, ULB, Programme Avenir des peuples des forêts tropicales, 2000.

21. Le terme «tribu » traduit ici le vocable qabîla, par lequel les Maures désignent leurs unités politico-généalogiques de référence, souvent associées à un territoire. En schématisant, la qabîla est un groupe de personnes plus ou moins large dont les membres se considèrent liés par des liens de parenté, de solidarité ou de sujétion renvoyant, au moins pour ce qui est du «noyau» (samîm) du groupe, à un ancêtre commun éponyme. Fondée idéalement sur l'équivalence de ses membres, qui se qualifient entre eux de "cousins » (azwlâd 'amm = «fils de l'oncle paternel»), la qabîla est aussi une structure hiérarchique où des "dépendants » (esclaves, anciens esclaves, artisans) sont associés à leurs " patrons » tribaux. Ils en sont solidaires, bon gré mal gré, pour la défense du territoire tribal commun, le règlement des compensations pour agression ou meurtre (diya)...

22. Lire J. Boulègue, La Sénégambie, du milieu du Xve siècle au début $d u$ XVII siècle, thèse de doctorat de $3^{\mathrm{e}}$ cycle, Université Paris-La Sorbonne, 1968, p. 90-96 ; A. W. Ould Cheikh, Nomadisme, islam et pouvoir politique dans la société maure précoloniale, thèse de doctorat, Université Paris 5, 1985 ; B. Barry, Le Royaume du Waalo, Paris, Maspéro, 1972; M. al-Mukhtâr al-Saad, Imârat al-Trârza wa 'alâqâtiha al-tijâriyya wa-l-siyyâsiyya ma'a Faransâ (1703-1860), thèse de doctorat, Université Tunis 1, 1999.

23. La société maure précoloniale était dominée par les groupes statutaires des hassân (guerriers) et des zwâya (marabouts), ces derniers subissant l'hégémonie politique 
des premiers. Les zwâya avaient une mainmise directe sur la terre et ses ressources, les guerriers se contentant d'y exercer leur autorité, notamment à travers le paiement de tributs comme ceux exigés des Imraguen.

24. A. Gruvel, Industries des pêches sur la côte occidentale de Mauritanie, Paris, Société d'éditions géographiques, maritimes et coloniales, 1927; Lt. Lotte, "Coutumes des Imraguen (Côte de Mauritanie, AOF) », Journal de la Société des Africanistes, tome VII, n 1, 1937, p. 41-48; P. Mercier et G. Balandier, «Émancipation par rachat des Imraguen sur le Banc d'Arguin et Cap Timiris (Mauritanie). Techniques et méthodes de pêche ", Notes africaines, $\mathrm{n}^{\circ} 33$, 1947, p. 22-34.

25. P. Mercier et G. Balandier, «Émancipation par rachat des Imraguen...", art. cit.

26. A. W. Ould Cheikh, L'Identité imraguen et la gestion locale de l'aire du PNBA. Approche historique et sociologique, Nouakchott, PNBA, 2002.

27. Lire P. Bonte, La Montagne de fer, Paris, Karthala, 2001. 28. M. L. Ould Dahi, M. L. Ould Baba et al., Le Littoral mauritanien, un patrimoine national, une ouverture sur le monde, Nouakchott, ministère des Pêches et de l'Économie maritime, 2004.

29. A. W. Ould Cheikh, Analyse des modes de régulation de l'accès aux ressources naturelles renouvelables. Aspects sociologiques, Nouakchott, PNBA, 2003.

30. Les Réserves de biosphère, qui forment un réseau mondial, ont été instituées par l'Unesco (programme «Homme et biosphère») pour promouvoir un environnement durable attentif aux liens entre conservation de la biodiversité et développement socioéconomique, et valorisant les liens entre diversité biologique et diversité culturelle.

31. P. Pélissier, Les Paysans du Sénégal. Les civilisations agraires du Cayor à la Casamance, Saint Yrieix, imp. Fabrègue, 1966. 32. V. Martin et C. Becker, «Documents pour servir à l'histoire des îles du Saloum ", Bulletin de l'Ifan, vol. 41, série B, n 4, 1979, p. 722-772.

33. Se reporter à G. E. Brooks, Landlords and Strangers. Ecology, Society and Trade in West Africa. 1000-1630, Boulder, Westview Press, 1993 et J. Boulègue, La Sénégambie..., op. cit., p. 150-171. 34. J. Boulègue, La Sénégambie..., op. cit., p. 96-121 et p. 249-270. 35. R. Van chi Bonnardel, «Exemples de migrations multiformes intégrées. Les migrations de Niominka (îles du Bas Saloum, Sénégal) », Bulletin de l'Ifan, vol. 39, série B, ${ }^{\circ} 4$, 1977, p. 837-889.

36. J.-P. Chauveau, «La pêche maritime artisanale dans le delta du Saloum », in J.-P. Chauveau et F. Laloë (dir.), L'Estuaire et la mangrove du Sine Saloum, Paris, Unesco, 1985.
37. F.-X. Pelletier, Les Imraguen, les hommes qui cueillent la vie, Paris, Flammarion, 1986.

38. A. W. Ould Cheikh, L'Identité imraguen..., op. cit.

39. É. Le Roy, «La loi sur le domaine national a vingt ans: joyeux anniversaire?», Mondes en développement, vol. 13, $\mathrm{n}^{\circ} 52,1985$, p. 667-685.

40. M.-C. Cormier-Salem, «Appropriation des ressources, enjeu foncier et espace halieutique sur le littoral ouestafricain », in J.-P. Chauveau, E. Jul-Larsen et C. Chaboud (dir.), Les Pêches piroguières..., op. cit., p. 205-229.

41. J.-P. Chauveau, op. cit, 2000.

42. Les populations lebou contrent la démarche d'autochtonisation des droits non par la négociation des règles d'accès, mais plutôt en consolidant leur présence foncière, comme à Djiffer. L'installation dans ce campement autrefois fréquenté par les seuls Niominka s'est faite progressivement: d'abord pour de courtes campagnes de pêche puis plus durablement avec la construction de maisons en dur. La croissance démographique des Lebou à Djiffer leur a donné du poids dans les joutes politiques locales. Ils disposent ainsi d'une influence sur le conseil rural qui leur permet de contourner les règles autochtones $\mathrm{d}$ 'installation et d'accès aux ressources.

43. Les îles bissau-guinéennes des Bijagos constituent un exemple de mise en œuvre par un État d'une régulation par l'autochtonie, bien qu'elle profite peu aux populations bijogos. Voir T. Dahou et al., "La gouvernance des aires marines protégées: leçons ouest-africaines ", VertigO, vol. 5, n³, 2004, sur < www.vertigo.uqam.ca >.

44. Des évolutions comparables sont observées au Cameroun. Voir P. Geschiere, «Ecology, belonging and xenophobia: the 1994 forest law in Cameroon and the issue of "community" ", in H. Englund et F. Nyamnjoh (eds), Rights and the Politics of Recognition in Africa, Londres, Zed Books, 2004, p. 237-259.

45. E. Hobsbawm et T. Ranger (eds), The Invention of Tradition, Cambridge, Cambridge University Press, 1983. 46. T. Murray Li, «Purification ethnique, savoirs récursifs et dilemmes du territorialisme», Revue internationale des Sciences sociales, $\mathrm{n}^{\circ} 173,2002$, p. 401-412.

47. M. Watts, «Contested communities, malignant markets, and gilded governance: justice, resource extraction and environmental justice in the tropics", in C. Zerner (ed.), People, Plant and Justice: the Politics of Nature Conservation, New York, Columbia University Press, 2000, p. 21-51.

48. P. Geschiere et F. Nyamnjoh, "Capitalism and autochthony: the seesaw of mobility and belonging", Public Culture, vol. 12, n 2, 2000, p. 423-452. 
Politique africaine

189 L'autochtonie dans les Aires marines protégées

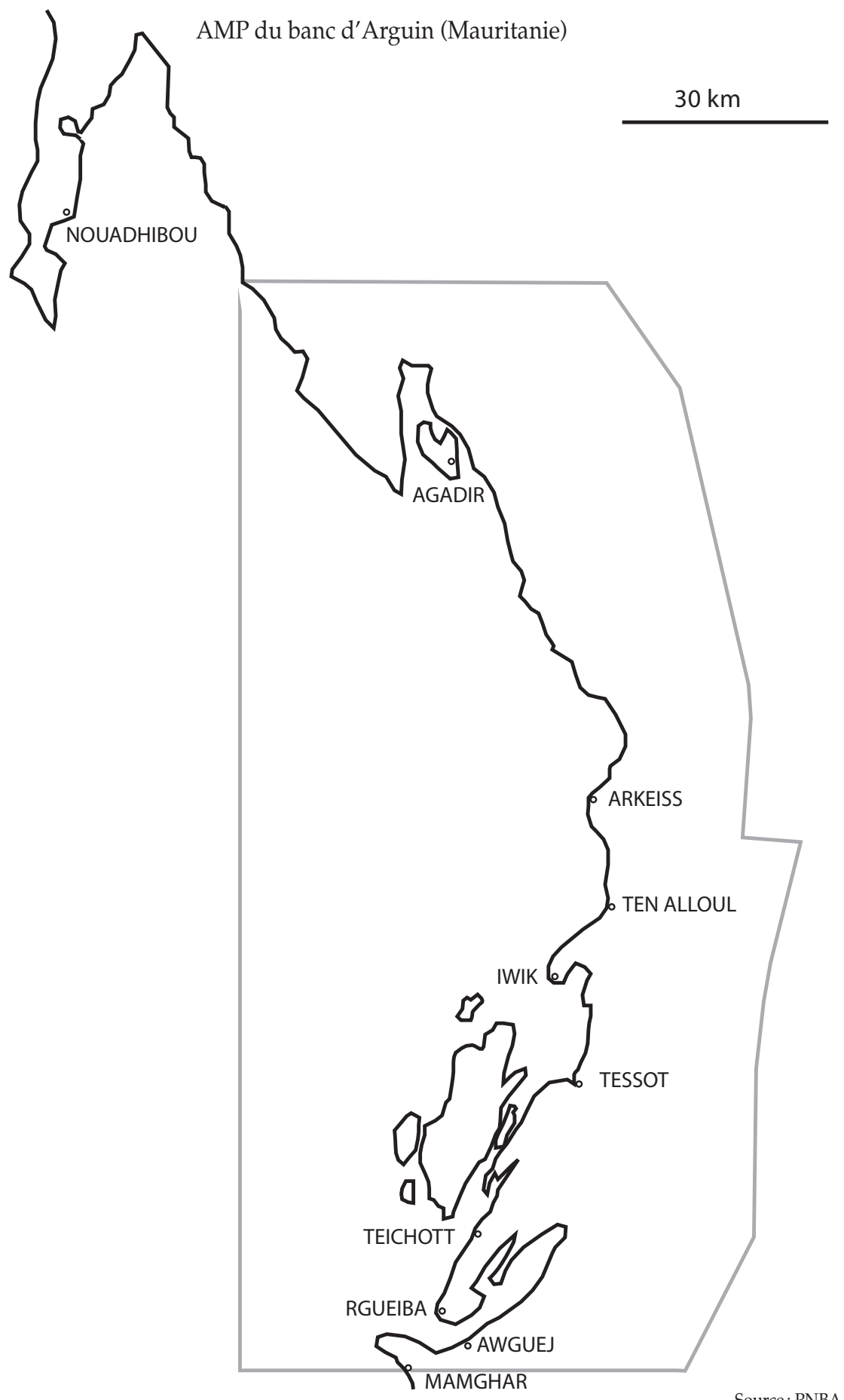




\begin{tabular}{|l|l|l|}
\hline Débat & Terrain & Documents \\
\hline
\end{tabular}

AMP du delta du Saloum (Sénégal)
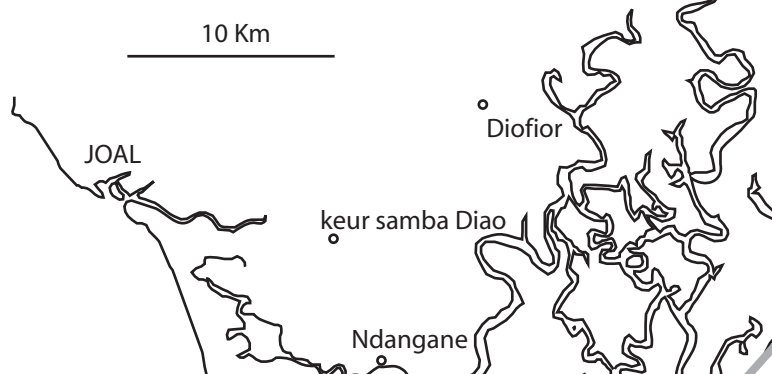

Djiffer
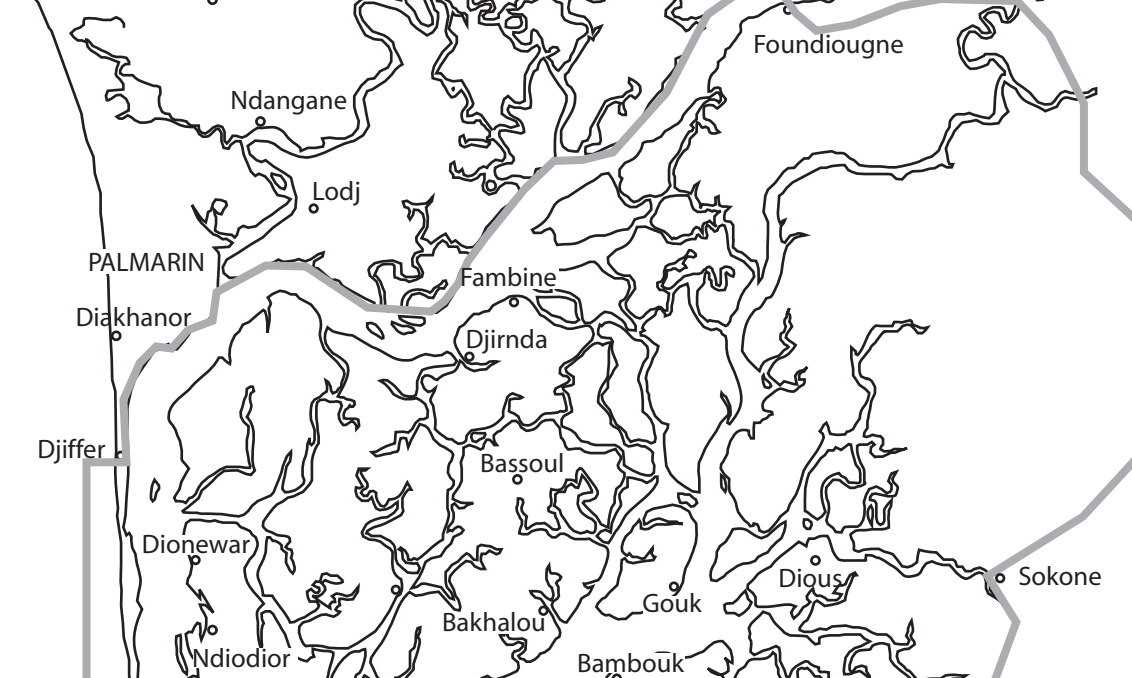